\title{
Gut microbiota trajectory in early life may predict development of celiac disease
}

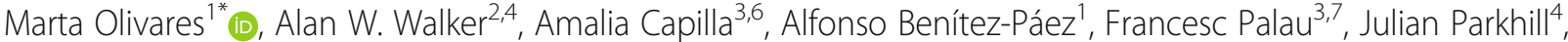 \\ Gemma Castillejo ${ }^{5}$ and Yolanda Sanz ${ }^{1}$
}

\begin{abstract}
Background: To investigate whether alterations in the developing intestinal microbiota and immune markers precede celiac disease (CD) onset in infants at familial risk of developing the disease.

Methods: A nested case-control study was carried out as part of a larger prospective cohort study, which included healthy full-term newborns (> 200) with at least one first relative with biopsy-verified CD. The present study includes cases of CD $(n=10)$ and the best-matched controls $(n=10)$ who did not develop the disease after 5 -year follow-up. Fecal microbiota, assessed by high-throughput $16 \mathrm{~S}$ rRNA gene amplicon sequencing, and immune parameters were profiled at 4 and 6 months of age and related to CD onset.

Results: The microbiota of infants who remained healthy showed an increase in bacterial diversity over time, characterized by increases in Firmicutes families, but not those who developed CD. Infants who subsequently developed CD showed a significant reduction in slgA levels over time, while those who remained healthy showed increases in TNF-a correlated to Bifidobacterium spp. An increased relative abundance of Bifidobacterium longum was associated with control children while increased proportions of Bifidobacterium breve and Enterococcus spp. were associated with CD development.
\end{abstract}

Conclusion: The findings suggest that alterations in the early trajectory of gut microbiota in infants at CD risk could influence the immune maturation process and predispose to CD, although larger population studies are warranted to confirm this hypothesis.

Keywords: Celiac disease, Intestinal microbiology, HLA genes

\section{Background}

Celiac disease (CD) is an immune-mediated systemic disorder elicited by an aberrant response to dietary gluten proteins found in wheat, rye, and barley, which develops in genetically predisposed individuals [1]. The disease is strongly associated with the human leukocyte antigen $H L A-D Q A 1$ and $H L A-D Q B 1$ genes expressed as surface heterodimers in antigen-presenting cells (APCs). The majority of CD patients $(95 \%)$ carry the HLADQ2.5 heterodimer, while most of the remaining patients have the HLA-DQ8 heterodimer [2]. Approximately $30-40 \%$ of the Caucasian subjects carry these risk haplotypes but most of them do not develop the disease,

\footnotetext{
* Correspondence: m.olivares@iata.csic.es

${ }^{1}$ Microbial Ecology, Nutrition and Health Research Unit, Institute of Agrochemistry and Food Technology, National Research Council (IATA-CSIC), C/Catedrático Agustín Escardino, 7. 46980, Paterna, Valencia, Spain Full list of author information is available at the end of the article
}

which has a prevalence rate of $1-3 \%$ in the general population and up to $10 \%$ in first-degree relatives of $C D$ patients $[3,4]$. Therefore, the $H L A-D Q 2 / D Q 8$ gene variants are necessary but not sufficient for the disease to develop. Although non- $H L A$ variants also contribute to CD risk [4], studies on the heritability of $\mathrm{CD}$ in twins indicate that genetics alone cannot explain $\mathrm{CD}$ onset and that nonshared environmental elements also contribute [5].

Gluten intake is the only environmental trigger of $\mathrm{CD}$ with an established pathogenic role. APCs recognize gluten peptides and activate lamina propria infiltrating $\mathrm{T}$ lymphocytes. This leads to the release of pro-inflammatory cytokines, mainly interferon (IFN) $\gamma$ and IL-15, activation of cytotoxic $\mathrm{T}$ cells, and profound tissue damage $[6,7]$. Exclusion of gluten from the diet is the only treatment available, but adherence to a gluten-free diet (GFD) can be difficult. Therefore, a better understanding of modifiable 
factors contributing to the breakdown of gluten tolerance would be desirable to enable novel strategies for primary prevention.

The timing of gluten introduction to the infant's diet may offer a window of opportunity for protection against $\mathrm{CD}$ in predisposed individuals $[8,9]$; however, it has not proven effective in recent randomized intervention studies (PreventCD and CELIPREV) [10, 11]. Epidemiological data suggest that additional environmental factors, such as type of delivery at birth, milk-feeding practices, intestinal infections, and/or use of antibiotics, could also determine CD risk [12-16]. A commonality of the aforementioned factors is that they impact on gut microbiota and, thereby, may influence its role in guiding the immune system towards development, or not, of gluten tolerance [17].

Observational studies have reported alterations in the composition of the microbiota of CD patients as well as increased abundance of virulence-related genes in intestinal pathobionts [18]. Furthermore, some authors reported that some of these alterations are not due to adherence to a GFD [19] and may be associated with gastrointestinal symptoms in untreated CD [20] and GFDtreated patients [21]. The contributing role of perturbations in the gut microbiota, and of specific enteric bacteria, to gluten-induced immunopathology has been proven in animal models [22]. Further evidence from longitudinal studies in human subjects is, however, needed to elucidate the role of the gut microbiota in CD etiology. With this aim, the prospective PROFICEL study enrolled a cohort of newborn healthy infants at familial risk of developing $\mathrm{CD}$ to monitor the progressive assembly of gut microbiota and its temporal relationship with factors potentially contributing to $\mathrm{CD}$ risk. Initial studies in this cohort of infants showed that the intestinal microbiota is influenced by both milk-feeding type and the HLA-DQ genotype [23, 24].

Building upon these findings, the present study aimed to investigate whether alterations in the developing intestinal microbiota and immune markers precede CD onset in infants at familial risk of developing the disease. The ultimate goal of the study is to shed light on the interactions between modifiable environmental and heritable factors in $\mathrm{CD}$ with a view to helping progress towards primary prevention.

\section{Methods}

\section{Study design and follow-up}

A nested case-control study was carried out as part of a larger prospective cohort study (PROFICEL), which included healthy full-term newborns with at least one first relative with biopsy-verified CD. Enrollment lasted from June 2006 to November 2010, as described elsewhere [23]. The present study includes cases of CD $(n=10)$ and the best-matched controls $(n=10)$ from the cohort who did not develop the disease by the time of cases' disease onset. Data regarding the mode of delivery, size, weight, and weeks of gestation were recorded at birth (Table 1). The health status, antibiotic intake, and feeding habits (i.e., breastfeeding and formula feeding, introduction of complementary foods) were monitored during the study period (5 years).

A total of 18 fecal samples from 9 controls and 9 CD cases were collected at 4 months, and a total number of 20 stool samples from 10 controls and 10 CD cases were collected at 6 months and analyzed in the present study. The DNA typing for CD HLA DQA1 and HLA DQB1 genes genotype was elucidated using sequence-specific primers (PCR-SSP) [23]. The HLA-DQ genotyping allowed the classification of the infants into three groups: the group 1 (high risk) included those individuals carrying the DQ2 haplotype in both cis (DQA1*05:01-DQB1*02:01 in homozygosis) and trans conformations (DQA1*02:01DQB1*02:02 with DQA1*05:05-DQB1*03:01 in heterozygosis), associated with the highest probability (20\%) of developing $\mathrm{CD}$. The group 2 (intermediate risk) included those infants carrying the DQ2 haplotype in cis conformation along with any other haplotype, as well as infants carrying the DQ8 haplotype (DQA1*03:01 DQB1*03:02) in homozygosis. This genotype is associated with a $7 \%$ probability of developing CD. The group 3 (low risk) included those infants with other common genotypes not associated with CD.

Ten infants were diagnosed with $\mathrm{CD}$ at the age shown in Table 1. The disease was diagnosed according to the 1990 criteria of the European Society of Pedriatric Gastroenterology, Hepatology and Nutrition (ESPGHAN) based on symptoms, positive anti-tissue transglutaminase (tTG) antibodies, quantified using a tTG-IgA ELISA kit (Pharmacia Diagnostics, $\mathrm{GmbH}$ ), and the histologic analysis of duodenal biopsy demonstrating villous atrophy compatible with Marsh 3 classification. From the same cohort, a subset of best-matched control children $(n=10)$ regarding the type of delivery, HLA-DQ genotype, and type of feeding were selected for comparative purposes. In both groups, exclusion criteria included the onset of other disorders related to immune dysregulation such as type-1 diabetes, allergies, and IgA deficiency.

\section{Fecal sample processing}

Fecal sample processing was done as described previously [24]. Briefly, fecal samples were diluted 1:10 $(w / v)$ with PBS (130 mM NaCl and $10 \mathrm{mM} \mathrm{Na}_{2} \mathrm{HPO}_{4}, \mathrm{pH} 7.4$ ) and homogenized. Aliquots submitted to low-spin centrifugation $\left(600 \mathrm{~g}, 2 \mathrm{~min}\right.$, at $\left.4{ }^{\circ} \mathrm{C}\right)$ were used for DNA extraction (FastDNA ${ }^{\mathrm{m}}$ SPIN kit for Soil, MP Biomedicals, Santa Ana, CA, USA). Aliquots submitted to high-speed centrifugation $\left(15,000 \mathrm{~g}, 10 \mathrm{~min}\right.$, at $\left.4{ }^{\circ} \mathrm{C}\right)$ were used for immune marker quantification. 
Table 1 Characteristics of the infants included in the study

\begin{tabular}{|c|c|c|c|c|c|c|c|c|c|c|c|c|}
\hline & ID & $\begin{array}{l}\text { Type of } \\
\text { delivery }\end{array}$ & $\begin{array}{l}{ }^{\text {aHLA-DQ }} \\
\text { genotype }\end{array}$ & $\begin{array}{l}\text { CD diagnosis } \\
\text { (months) }\end{array}$ & Gender & $\begin{array}{l}\text { Relatives } \\
\text { with CD }\end{array}$ & $\begin{array}{l}\text { Gestation } \\
\text { (weeks) }\end{array}$ & $\begin{array}{l}\text { Height }(\mathrm{cm}) \\
\text { at birth }\end{array}$ & $\begin{array}{l}\text { Weight (g) } \\
\text { at birth }\end{array}$ & $\begin{array}{l}\text { Type of feeding } \\
\text { at } 4 \text { months }\end{array}$ & $\begin{array}{l}\text { Type of feeding } \\
\text { at } 6 \text { months }\end{array}$ & $\begin{array}{l}\text { Intake of } \\
\text { antibiotics }\end{array}$ \\
\hline \multirow{10}{*}{$\begin{array}{l}\text { Controls } \\
(n=10)\end{array}$} & $\mathrm{Cl}$ & Vaginal & 1 & & $F$ & Brother & 40 & 52 & 3730 & FF & $C+F F$ & \\
\hline & $C 2$ & Vaginal & 2 & & M & Brother & 40 & 51 & 3750 & $\mathrm{FF}$ & $C+F F$ & \\
\hline & C3 & Vaginal & 2 & & F & Father & 40 & 50 & 3335 & $\mathrm{BF}$ & $C+B F$ & \\
\hline & C4 & Vaginal & 1 & & F & Mother & 39 & 48 & 2960 & $\mathrm{BF}$ & $C+F F+B F$ & \\
\hline & $C 5$ & Cesarean & 1 & & M & Mother & 38 & 50 & 2800 & $\mathrm{BF}$ & $C+B F$ & Clarithromycin \\
\hline & C6 & Vaginal & 2 & & F & Mother & 39 & 49 & 2900 & $\mathrm{FF}$ & $C+F F$ & \\
\hline & $\mathrm{C} 7$ & Cesarean & 2 & & M & Sister & 38 & 51 & 3190 & $\mathrm{BF}$ & $C+F F+B F$ & \\
\hline & $\mathrm{C} 8$ & Vaginal & 1 & & $\mathrm{~F}$ & Father & 39 & 49 & 3890 & $\mathrm{BF}$ & $C+B F$ & \\
\hline & C9 & Vaginal & 2 & & $\mathrm{~F}$ & Bother & 38 & 50 & 3610 & $\mathrm{BF}$ & $C+B F$ & \\
\hline & $\mathrm{C} 10^{\mathrm{d}}$ & Vaginal & 3 & & M & Mother & 40 & 52 & 3880 & $\mathrm{BF}$ & $C+B F$ & \\
\hline${ }^{b}$ Mean (SE) & & & & & & & $39.1(0.3)$ & $50.2(0.42)$ & $\begin{array}{l}3405 \\
(133.2)\end{array}$ & & & \\
\hline \multirow{10}{*}{$\begin{array}{l}\text { CD cases } \\
(n=10)\end{array}$} & CD1 & Vaginal & 1 & 40 & $\mathrm{~F}$ & Mother & 36 & 51 & 4890 & $\mathrm{FF}$ & $C+F F$ & \\
\hline & CD2 & Vaginal & 2 & 30 & M & Sister & 40 & 50 & 3450 & $\mathrm{FF}$ & $C+F F$ & Augmentin \\
\hline & CD3 & Vaginal & 2 & 21 & M & Brother & 37 & 48 & 3380 & $\mathrm{BF}$ & $C+S M$ & Amoxicillin \\
\hline & CD4 & Vaginal & 1 & 27 & $\mathrm{~F}$ & Mother & 38 & 48 & 3005 & $\mathrm{BF}$ & $C+F F$ & \\
\hline & CD5 & Cesarean & 1 & 16 & $\mathrm{~F}$ & Sister & 37 & 47 & 2730 & $\mathrm{BF}$ & $C+F F$ & Amoxicillin \\
\hline & CD6 & Vaginal & 2 & 26 & $F$ & Mother & 39 & 49 & 3200 & $\mathrm{FF}$ & $C+F F$ & Augmentin \\
\hline & CD7 & Cesarean & 1 & 36 & $F$ & Mother & 38 & 50 & 4360 & $\mathrm{BF}$ & $C+B F$ & \\
\hline & CD8 & Vaginal & 1 & 24 & M & $\begin{array}{l}\text { Mother + } \\
\text { sister }\end{array}$ & 41 & 50 & 4170 & $\mathrm{BF}$ & $C+S M$ & Augmentin \\
\hline & CD9 & Vaginal & 2 & 82 & M & $\begin{array}{l}\text { Mother + } \\
\text { sister }\end{array}$ & 37 & 48 & 3030 & $\mathrm{BF}$ & $C+B F$ & \\
\hline & $\mathrm{CD} 10^{\mathrm{d}}$ & Vaginal & 2 & 40 & $\mathrm{~F}$ & Mother & 40 & 47 & 3070 & $\mathrm{BF}$ & $C+B F$ & \\
\hline${ }^{b}$ Mean (SE) & & & & $34.2(5.9)$ & & & $38.3(0.5)$ & $48.8(0.44)$ & $\begin{array}{l}3529 \\
(222.7)\end{array}$ & & & \\
\hline${ }^{c} p$ value & & 1.000 & 0.828 & - & 1.000 & 0.258 & 0.190 & 0.033 & 0.6385 & 1.000 & 0.172 & 0.143 \\
\hline
\end{tabular}

$B F$ breastfeeding, FF formula feeding, C complementary feeding (foods other than milk and water), SM soya milk

aGenetic risk of developing CD was established according to the HLA-DQ genotype (see the "Methods" section for details)

${ }^{b}$ Data of continuous variables are expressed as mean and standard error of the mean (in brackets)

'Statistically significant differences between cases and controls were established by applying using a $t$ test for continuous variables and the chi-square test for categorical variables at $p<0.050$

${ }^{\mathrm{d}}$ No microbiota analysis available for these samples at 4 months

\section{Immune marker quantification}

Calprotectin was quantified by diluting fecal samples in the calprotectin sample solution and using the ELISA Quantitative Fecal Calprotectin kit (DIAsource, Louvainla-Neuve, Belgium). Secretory $\operatorname{IgA}(\operatorname{sgA})$ and cytokines (tumor necrosis factor (TNF)- $\alpha$, interferon (IFN)- $\gamma$, interleukin (IL)-1 $\beta$ and IL-6) were quantified using ELISA commercial kits (Bethyl, Montgomery, TX, USA) and Max Deluxe set (Biolegend, San Diego, CA, USA) respectively. The threshold sensitivity level for sIgA was $7.8 \mathrm{ng} / \mathrm{mL}$; for TNF- $\alpha$, IFN- $\gamma$, and IL-6 was $7.8 \mathrm{pg} / \mathrm{mL}$; and for IL-1 $\beta$ was $2.0 \mathrm{pg} / \mathrm{mL}$.

\section{Sequencing of 16S rRNA gene amplicons}

The V1-V2 variable region of the bacterial 16S rRNA gene was amplified from the fecal DNA by PCR as described previously [25] and sequenced using the Illumina MiSeq platform $(2 \times 250$ bp reads $)$.

\section{Sequence analysis}

The 16S rRNA gene sequence data were processed using the Mothur software package [26] as described previously [25]. After filtering to remove poor quality sequences and chimeras, each sample was rarefied to 2698 reads to ensure equal sequencing depth for all subsequent comparisons. Good's coverage estimates at this sequencing depth averaged 99.6\% (minimum of 99.2\%). Clusters were analyzed using the Jaccard calculator, based on the presence/absence of operational taxonomic units (OTUs), and the Bray-Curtis calculator, based upon the presence/absence of OTUs and also on the relative abundance of each OTU. Diversity indexes (Shannon and inverse Simpson) were calculated using Mothur. OTUs were classified taxonomically by matching against the reference RDP database provided at the Mothur website (https://www.mothur.org/wiki/RDP_re ference_files\#Version_10). Classification of selected 
OTUs was verified using MegaBLAST against the NCBI database.

\section{Statistical analyses}

All data analyzed in this study are shown in Additional files 1 and 2: Tables S1 and S2. Data distribution was assessed with the Shapiro-Wilk $W$ test (SPSS software V19). Of the demographic data, the categorical variables were analyzed using the chi-square test (GradPad, 6.0) and the continuous variables (size, weight, and weeks of gestation at birth) using a $t$ test (SPSS software V19). Comparisons of data of immune parameters between groups were done using the $t$ test for non-paired samples and within groups over time were done using the $t$ test for paired samples (SPSS software V19). Compositional comparisons at the OTU, genus, family, and phylum levels were carried out using metastats [27] and LEfSe [28]. False discovery rate was used for $p$ value correction upon multiple comparisons using the Benjamini-Hochberg method [29]. With metastats, the relative abundance of each OTU was compared across the two populations (CD and control) by computing a two-sample $t$ statistic. The null distribution of $t$ was estimated non-parametrically using a permutation test [27]. For diversity measures, the Mann Whitney $U$ test was used for comparisons between infant groups and the Wilcoxon signed-rank test for comparisons within groups at different ages using R v3.12. Correlations between the microbiota composition and immunological parameters were established using the Spearman coefficient (GradPad, 6.0). Statistically significant differences were established at $p<0.05$.

\section{Results}

Demographic data, genetics, and early feeding pattern of the infants

A nested case-control study was conducted to investigate the role of the evolution of the infant gut microbiota in CD onset in a subset of subjects selected from a larger prospective cohort study [23]. The Table 1 shows the baseline characteristics of the infants included in the study. These were similarly distributed between the two groups $(p<0.05)$, except for height at birth, which was higher in the controls than in the CD group (50.2 versus $48.8 \mathrm{~cm}, p<0.05)$. Regarding the type of feeding, at 4 months of age, 3 subjects were fed with formula and 7 were breast-fed in both the control and $C D$ group. According to pediatricians' recommendations, all infants started the transition from milk feeding to the first solid foods between 4 and 6 months of age. The introduction of gluten was initiated from 6 months of age onwards in all cases. From birth to 4 months of age, no infections or antibiotic intake were registered. Up to 6 months, a total of 6 infants suffered from infections during the time frame of our study and were treated with antibiotics. Infant CD2 suffered from pharyngitis; CD3 suffered from an upper respiratory tract infection; CD5 suffered from otitis; CD6 and CD8 suffered from an upper respiratory tract infection and also from otitis, and control C5 suffered from bronchitis. No significant differences $(p=0.143)$ were detected between groups in the intake of antibiotics.

The diagnosis of $\mathrm{CD}$ for most cases occurred between the ages of 16-40 months (mean 34.2 months, SE 5.9), with the exception of one case (CD9), who was diagnosed at 82 months of age. At the time of diagnosis, all CD children had elevated levels of tTG antibodies and the following symptomatology: four cases presented diarrhea and abdominal distension, one constipation and asthenia, and five had no symptoms.

\section{Early evolution of gut microbiota in relation to $C D$ development}

Overall cluster analysis of the 16S rRNA gene sequence data, using either the Jaccard or the Bray-Curtis calculators, showed there was little distinct clustering of the early microbiota profiles by disease status later in life (Fig. 1). However, the parsimony test as implemented in Mothur software [26] suggested that there was a borderline significant difference in bacterial community membership (Jaccard) $(p=0.045)$, but not in the community structure (Bray-Curtis) at 4 months of age between the two groups of infants. This significance disappeared by 6 months $(p=0.640)$.

Compositional analysis revealed the presence of eight phyla across the whole dataset, but only four of them were above the mean value of $1 \%$ of the total number of reads. The relative abundance of reads corresponding to Bacteroidetes, Proteobacteria, Firmicutes, and Actinobacteria in the CD and control groups at 4 and 6 months are represented in Fig. 2. At 4 months of age, children that later developed CD harbored a significantly higher proportion $(p=0.036)$ of Firmicutes compared to those that remained healthy (control group). This generally occurred alongside proportional reductions in Proteobacteria and Bacteroidetes, although the differences in these two phyla were not significant. At 6 months, no differences at the phylum level were observed between the two groups of children (Fig. 2).

We also evaluated the possible influence of covariates on gut microbiota composition, which could bias the results of comparisons between infants that remained healthy and those who developed $\mathrm{CD}$ later in life. No differences $(p>0.05)$ depending on the HLA-DQ genotype (high risk versus intermediate risk), the type of milk feeding (breast milk versus formula together or not with complementary feeding), and the effect of antibiotic intake in the samples collected at 6 months of age (positive 

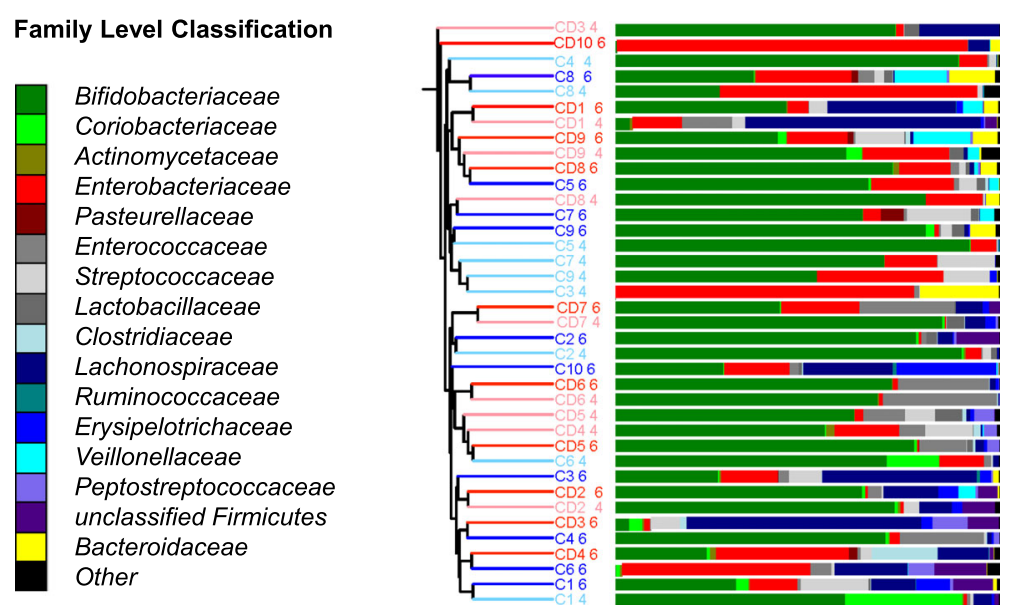

Fig. 1 Jaccard dendrogram colored by disease status, with controls at 4 months (dark blue), controls at 6 months (light blue), and children who developed CD (CD group) at 4 months (pink) and at 6 months (red)

intake versus negative intake) were detected with metastats after correcting for multiple comparisons.

The early microbiota of $\mathrm{CD}$ children and the control group showed a different developmental process regarding the relative abundance of Firmicutes. In control children, Firmicutes proportions showed a statistically significant increase from 4 to 6 months of age $(p=0.011)$, while in the CD children, differences were not detected over time (Fig. 2). At both 4 and 6 months, no differences were observed between groups of children in the most abundant bacterial families and genera. When considering the gut microbiota development over time within groups, children that remained healthy showed proportional increases in Enterococcaceae $(p=0.030)$ (Fig. 3a) and in

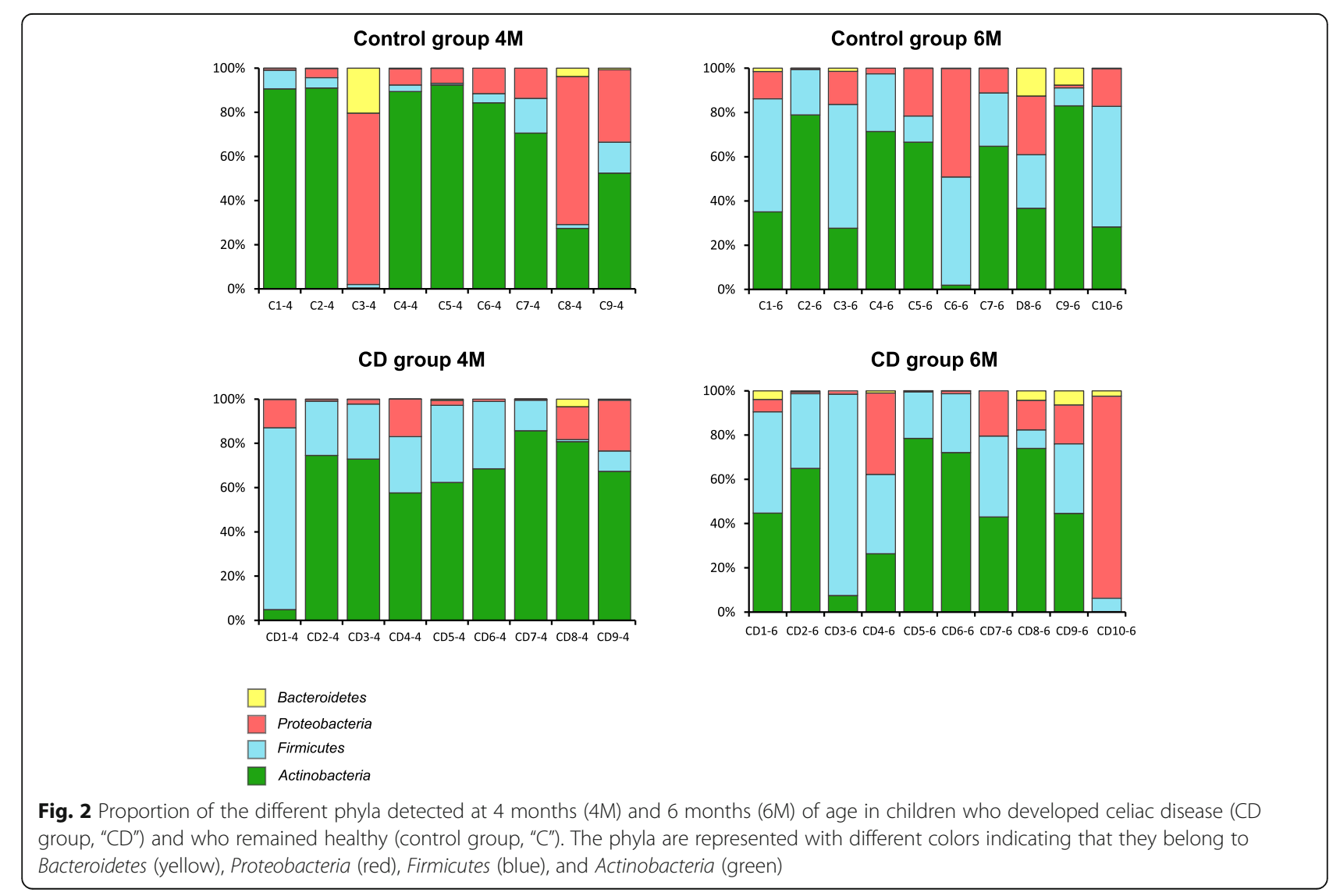



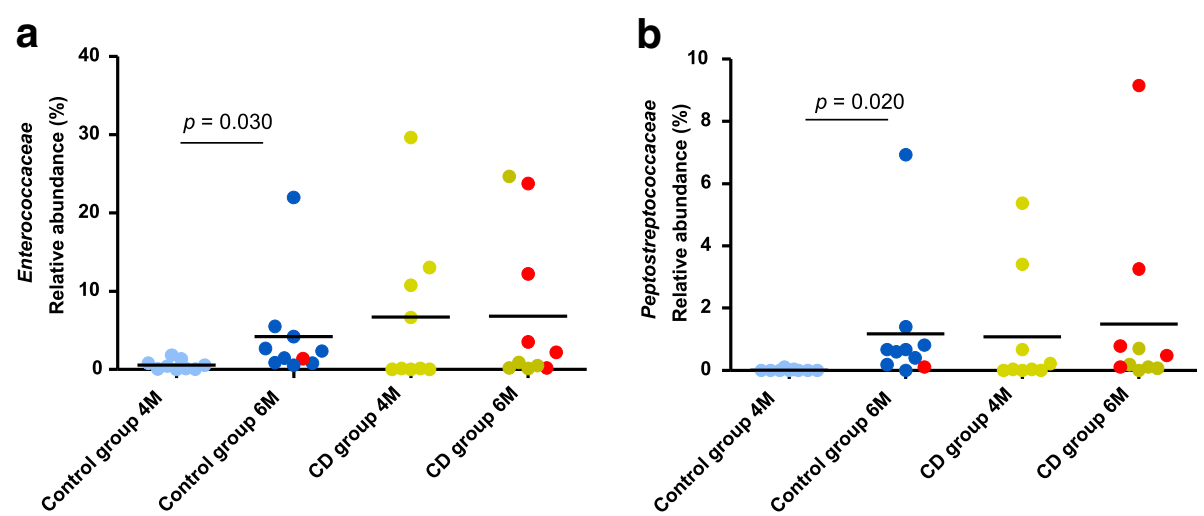

Fig. 3 a, b Significantly different families detected at 4 months (4M) and 6 months $(6 \mathrm{M})$ of age in children who developed celiac disease (CD group) and who remained healthy (control group). Statistically significant differences were established at $p<0.05$. Red dots correspond to the infants that were exposed to antibiotics from 4 to 6 months of age

Peptostreptococcaceae $(p=0.020)$ (Fig. 3b) from 4 to 6 months of age, whereas no differences were found in the CD group over time.

We also used the LEfSe software program to detect putative bacterial biomarker species that could predict later development of CD [28]. At the level of the individual OTUs (a total of 374-see Additional file 1: Table S1), LEfSe analyses indicated that increased relative abundance of Bifidobacterium longum was associated with the control group (Linear Discriminant Analysis (LDA) $=5.004, p=0.021$ ), while that of Bifidobacterium breve $(\mathrm{LDA}=4.846, p=0.032)$ and Enterococcus spp. (Enterococcus gallinarum/casseliflavus/faecium, LDA $=4.507, p=0.018$ ) were associated with the $\mathrm{CD}$ cases. The presence of Clostridium innocuum (LDA $=4.005, p=0.047$ ), Veillonella dispar/parvula LDA $=3.867$, $p=0.009$ ), and Intestinibacter bartlettii (LDA $=3.415$, $p=0.003$ ) were associated with the age of 6 months.

\section{Richness and diversity analyses}

Microbial diversity was analyzed in both groups at 4 and 6 months (Fig. 4). The control group that remained healthy showed a statistically significant increase in both richness and diversity over the study period, as measured by the number of observed OTUs $(p=0.024)$ (Fig. 4a), the Shannon diversity index $(p=0.009)$ (Fig. $4 \mathrm{~b})$, and the inverse Simpson diversity index ( $p=0.013$ ) (Fig. 4c). In contrast, there was no significant increase in the microbial diversity over the study period in the group of children who developed CD later in life $(p=0.155, p=0.193$, and $p=0.407$, for comparisons of number of OTUs, Shannon, and inverse Simpson indexes, respectively).

\section{Quantification of immune markers}

Children who developed CD showed a significant reduction $(p=0.037)$ in fecal sIgA levels from 4 to 6 months of age, while the reductions did not reach statistical significance in children that remained healthy (Fig. 5a). No differences in the calprotectin levels were observed between groups at 4 months or 6 months or within groups over the study period (Fig. 5b). There was huge variability in calprotectin levels between individuals, which was more pronounced in the case of the CD children. In this group, the coefficient of variation $(\mathrm{CV})$ was $85.0 \%$ at 4 months, and $103.2 \%$ at 6 months.

Regarding differences in cytokines, children who developed CD had higher levels of intestinal IL-6 ( $p=$ 0.043 ) than the control group at 4 months, but this difference disappeared at 6 months (Fig. 6a). The concentration of TNF- $\alpha$ tended to increase over time only in control children ( $p=0.051$; Fig. $6 \mathrm{~b})$, which might be linked to maturation of the gut microbiota and its increased diversity. Comparisons between groups also revealed that the control group showed higher TNF- $\alpha$ levels than the CD group $(p=0.028)$ at 6 months (Fig. 6b). No differences were observed in the IL-1 $\beta$ or IFN- $\gamma$ levels between or within groups (Fig. 6c, d).

At 4 months, sIgA concentrations were positively correlated with Bacteroidaceae $(r=0.778, p=0.017)$ and Enterobacteriaceae $(r=0.733, p=0.031)$ and negatively with Lachnospiraceae $(r=-0.895, p=0.002)$ and Coriobacteriaceae $(r=-0.712, p=0.037)$ in the control group. Besides, a positive association was found between Firmicutes and calprotectin $(r=0.857, p=0.024)$. At 6 months, the control group showed a positive correlation between Bifidobacteriaceae and TNF- $\alpha(r=0.730, p=0.020)$ and two negative ones between "unclassified Lachnospiraceae" and IFN- $\gamma$ levels $(r=-0.644, p=0.049)$ and Bacteroidaceae and IFN- $\gamma$ levels $(r=-0.831, p=0.005)$. This latter negative correlation was also found in the CD group at the same time point $(r=-0.806, p=0.007)$.

\section{Discussion}

This is the first study to identify features of the bacterial community assembly and intestinal immune milieu that 

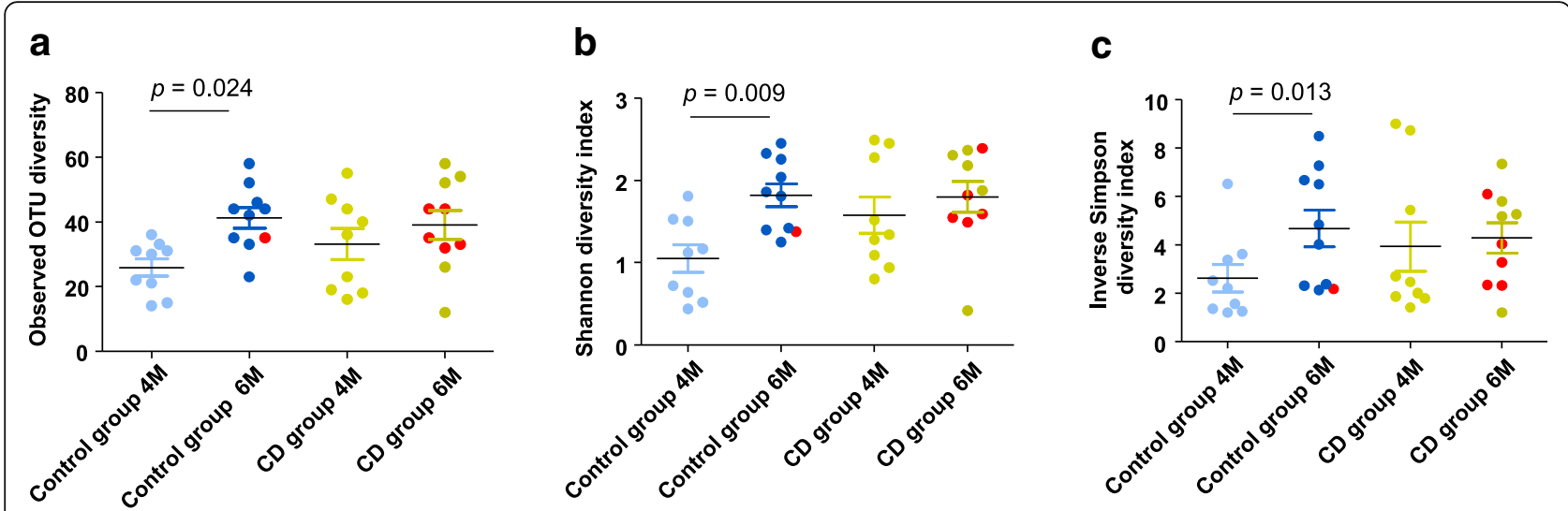

Fig. 4 Number of observed OTUs (a), Shannon (b), and the inverse Simpson (c) diversity indexes detected at 4 months (4M) and 6 months (6M) of age in children who developed celiac disease (CD group) and who remained healthy (control group). Values are expressed as means and standard error of means. Statistically significant differences were established at $p<0.05$. Red dots correspond to the infants that were exposed to antibiotics from 4 to 6 months of age

precede $\mathrm{CD}$ onset and that may constitute predictive markers of disease risk and modifiable risk factors. Although there were not great differences in microbiota composition between the two infant groups (those who later developed CD or those who remained healthy), specific differences were identified in the early evolution of bacterial communities and of immune markers associated with subsequent $\mathrm{CD}$ development.

Our study shows a significant increase in microbiota diversity of control children between the ages of 4 and 6 months, which did not occur in the child group who went on to develop CD. Similarly, specific bacterial communities followed a different trajectory over time in the study groups, revealing differences in the phylum Firmicutes, and the families Enterococcaceae and Peptostreptococcaceae belonging to this phylum, whose proportions increased from 4 to 6 months only in those children who did not develop CD. Previous studies reported that the phylogenetic diversity of the microbiota in the first stages of life increases over time [30-32]. It was also estimated that the Gini-Simpson $\alpha$-diversity index increases by 0.008 per day on average during the first month of life [33]. However, in the child group who went on to develop $\mathrm{CD}$, diversity did not increase significantly over time, showing an uncommon developmental pattern ("premature maturation") compared to the healthy group, since they started with a higher basal diversity level. Therefore, a progressive increase in microbial diversity seems to be a feature of the evolution of a healthy microbiota, which may increase resilience to CD. A previous prospective study with only one case developing $C D$ at 24 months of age also reported reductions in bacterial richness before disease onset ( 6 to 10 months of age) [34]. However, the findings of the
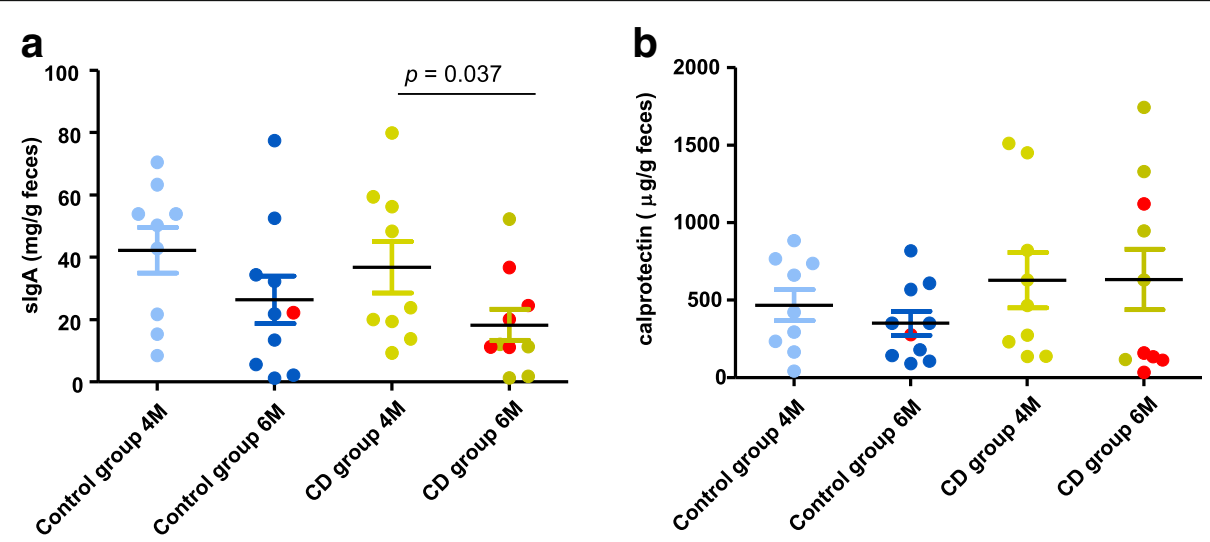

Fig. 5 Secretory $\lg A(\operatorname{sg} A)(\mathbf{a})$ and calprotectin (b) in feces collected at 4 months (4M) and 6 months (6M) of age from children who developed celiac disease (CD group) and who remained healthy (control group). Values are expressed as means and standard error of means. Statistically significant differences were established at $p<0.05$. Red dots correspond to the infants that were exposed to antibiotics from 4 to 6 months of age 

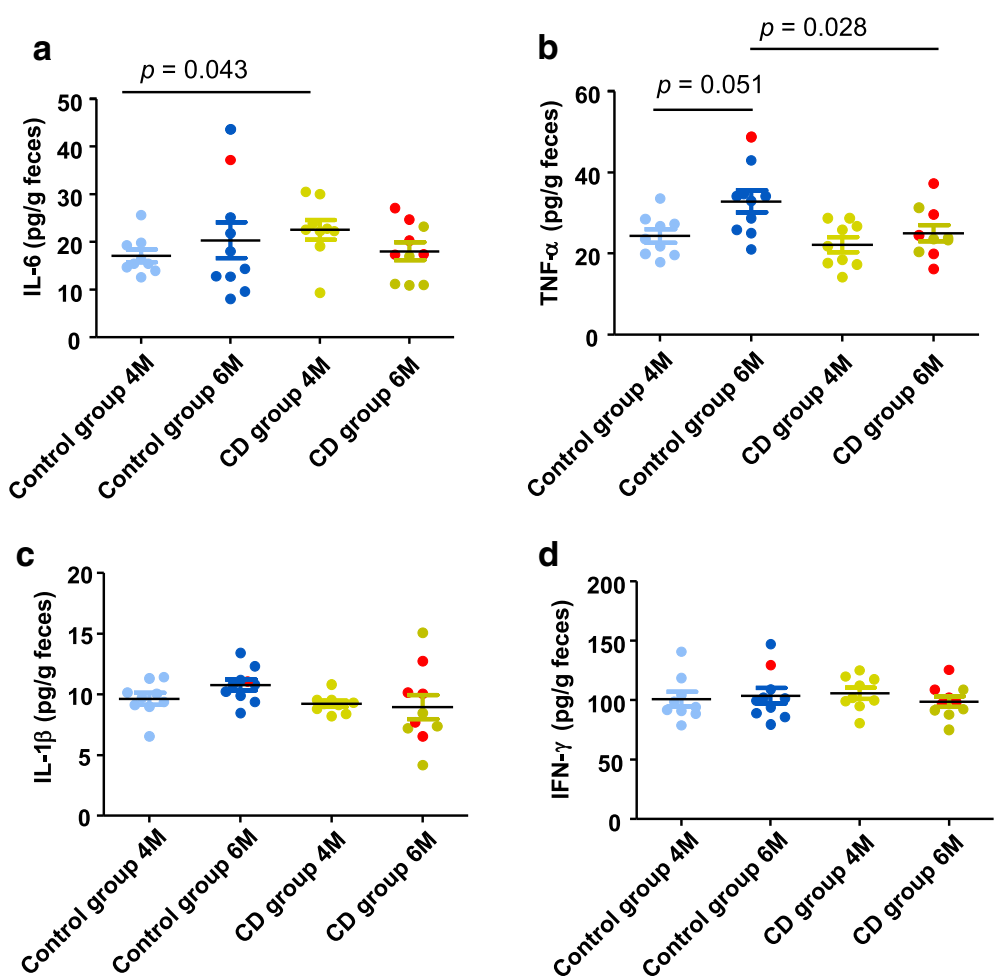

Fig. 6 Cytokine levels, interleukin (IL)-6 (a), tumor necrosis factor (TNF)-a (b), IL-1ß (c), and interferon (IFN)- $\gamma$ (d) in feces collected at 4 months $(4 \mathrm{M})$ and 6 months $(6 \mathrm{M})$ of age from children who developed celiac disease (CD group) and who remained healthy (control group). Values are expressed as means and standard error of means. Statistically significant differences were established at $p<0.05$. Red dots correspond to the infants that were exposed to antibiotics from 4 to 6 months of age

aforementioned study are of limited value since statistical analysis could not be performed with a single CD case. Furthermore, the results are incomparable since the cited study aimed to investigate how CD onset was affected by the time the gluten was introduced into the diet, introducing different variables from those considered in our study.

A number of observational and to a lesser extent intervention studies in adults have generally associated an increased bacterial diversity with a healthy phenotype [35-37]. The present study, however, suggests that a timely maturation of the gut microbiota towards one of higher complexity and diversity might also be required for proper development of immune tolerance in the early stages of life and that premature maturation and exposure to a complex microbiota may increase disease risk.

In our study, a control group of infants matching the disease group regarding the type of milk feeding, mode of delivery, and the HLA genotype was selected for comparative purposes. Therefore, the early increase in diversity observed in the infants who subsequently developed $\mathrm{CD}$ could be due to other uncontrolled environmental exposures that influence colonization waves of the infant's gut (perinatal factors, hospital and home environment, number of siblings, etc.) or additional genetic factors that create permissive conditions to accommodate a more heterogeneous microbiota. Although the effect of host genetics on the microbiota is less known than the effects of environmental factors, associations between the microbiome and genes related to innate immunity that could control gut colonization (e.g., pattern recognition receptors) have been identified through genome-wide association studies [38].

Intestinal infections and their treatment with antibiotics have been associated with an increased risk of developing $\mathrm{CD}$ in some studies [13]. However, our study infants only suffered from otitis and respiratory tract infections during the study period and the antibiotic treatment was not significantly associated with $C D$ onset. A possible impact of antibiotic intake on the microbiota of our infants could not be completely disregarded due to the relatively small size of the study. Nonetheless, the microbiota of those infants who later developed $C D$ already presented a pattern of maturation significantly different to the control group at 4 months of age (before the intake of antibiotics), according to the differences in the bacterial community membership (Jaccard similarity index) that were attributed to increases in Firmicutes.

Taking into account the $\mathrm{CD}$ genetic risk classification of the infants included in the study, we did not find 
significant association with the intestinal microbiota composition either. These results are in agreement with our previous studies reporting no major differences in microbiota composition as a function of genotype when comparing risk groups 1 and 2 by qPCR [23]. Notwithstanding, we did find differences when comparing the high-risk groups 1 and 2 with the low-genetic risk group 3 [23] and also when comparing the highest risk infants (group 1) with low-risk infants (group 3) [24].

In our previous studies on $\mathrm{CD}$, we found that the highrisk genotype for developing $\mathrm{CD}$ was associated with reduced numbers of Bifidobacterium (Actinobacteria phylum), specifically of the species $B$. longum [23] compared to the low-risk genotype. Accordingly, at that time, we hypothesized that microbiota differences associated with the HLA-DQ2/8 genotype could constitute an additional risk factor for $\mathrm{CD}$ onset. The present results further support the hypothesis that a reduced abundance of $B$. longum, dependent on both genetic and environmental factors, may constitute a risk factor for CD development and constitute an early predictive biomarker of $\mathrm{CD}$.

Our present study also suggests that early proportions of Firmicutes and members of the Actinobacteria phylum (B. longum) in infants at familial risk of developing $C D$ are influenced not only by the HLA-DQ2/8 genotype [23], but also possibly by other genetic and environmental factors. However, considering the complexity of the Firmicutes phylum, larger cohort sizes and indepth taxonomic studies will be needed to understand the potential biological role played by specific components of this phylum and their role in $\mathrm{CD}$ onset in response to other environmental factors.

Interestingly, we also observed a faster reduction in sIgA fecal levels in the children who went on to develop $\mathrm{CD}$ over time compared to healthy ones. sIgA is generally considered to act as a first line of defense in the intestine by interacting with intestinal antigens and microbes, preventing their penetration into the lamina propria, and therefore contributing to immunological homeostasis [39]. sIgA is also thought to play a role in mucosal sensing and control of commensal bacteria colonizing the gut [40]. Besides, sIgA influences the development of dendritic cells with a tolerogenic phenotype, which play a key role in maintaining intestinal homeostasis $[41,42]$. Thus, it can be hypothesized that a premature reduction of sIgA levels in the group of children that developed $\mathrm{CD}$ could be related to shifts in bacterial community development which impact the maturation of the mucosal immune functions, possibly increasing the risk of developing autoimmune dysfunctions. Moreover, the fact that $\mathrm{CD}$ frequently occurs in subjects with selective IgA deficiency has been interpreted as an indicator that there is a reduction in the infant's mucosal protection [6].
The differential profiles of fecal cytokines over time in the two groups of infants may also reflect differences in gut microbiota maturation. Early increased levels of IL-6 could be related to premature maturation of the microbiota of infants who finally developed $C D$, while increased levels of TNF- $\alpha$ at 6 months could reflect the host response to a more diverse microbiota acquired at this age by infants who remained healthy, and to its specific components such as Bifidobacterium, which showed a positive correlation with TNF- $\alpha$.

The strength of this report lies in the exhaustive prospective follow-up of the study cohort, where bias that usually affects retrospective studies has been excluded. Furthermore, a nested case-control study has integrated genotypic, dietary, immune, and microbiota data from biopsy-verified CD cases for the first time. The main limitation is the relatively small population size of the prospective study, taking into account the low prevalence of CD in this at-risk population $(\sim 10 \%)$. This limits the statistical power to investigate the effects of different covariates, including the possible role of antibiotics, which would have helped to reach more robust conclusions.

\section{Conclusions}

Overall, we demonstrate shifts in the early trajectory of the gut microbiota along with changes in immune markers in infants who later develop $\mathrm{CD}$. These alterations are indicative of deviations of the normal microbiota maturation process, which precede CD development. Further studies that build on these observations are warranted to progress in the understanding of the specificities of these deviations and the mechanisms by which they might increase disease risk in response to environmental triggers.

\section{Additional files}

Additional file 1: Table S1. Full list of OTUs and taxonomic classifications. (XLSX $87 \mathrm{~kb})$

Additional file 2: Table S2. Ecological descriptors and immune parameters. (XLSX $12 \mathrm{~kb}$ )

\section{Abbreviations \\ APCs: Antigen-presenting cells; CD: Celiac disease; ESPGHAN: European Society of Pedriatric Gastroenterology, Hepatology and Nutrition; GFD: Gluten-free diet; HLA: Human leukocyte antigen; IFN: Interferon; IL: Interleukin; OTUs: Operational taxonomic units; slgA: Secretory IgA; TNF: Tumor necrosis factor; tTG: Tissue transglutaminase}

\section{Acknowledgements}

We thank the families of the infants enrolled in the study for their commitment with the project. We thank the Wellcome Trust Sanger Institute's core sequencing team for carrying out the Illumina MiSeq sequencing of $16 \mathrm{~S}$ rRNA genes.

\section{Funding}

This work was supported by grants AGL2011-25169, AGL2014-52101-P, and AGL2007-66126-C03-03/ALI (YS and FP) from the Spanish Ministry of Economy and Competitiveness (MINECO). Funding for AWW and JP and 16S rRNA gene 
sequencing was provided by Wellcome Trust (Grant 098051); AWW and The Rowett Institute, University of Aberdeen, receive core funding support from the Scottish Government Rural and Environmental Science and Analysis Service (RESAS). The scholarship to MO from CSIC (JAEpre) and the contract to ABP from the European Union's Seventh Framework Program under the grant agreement no 613979 (MyNewGut) are also fully acknowledged.

\section{Availability of data and materials}

All data analyzed during this study are included in this published article (Additional files 1 and 2: Tables S1 and S2). The fasta files containing raw data from all samples and time points could be accessed at the European Nucleotide Archive (ENA) under project accession PRJEB23313 (samples ERS1993272 to ERS1993309).

\section{Authors' contributions}

YS conceived the study design; GC recruited and followed up the children AC and FP genotyped the infants; MO performed the DNA extractions and all immunological analyses; AWW performed the sequencing analysis; JP provided the critical resources, guidance, and support; MO, AWW, and ABP contributed to the data analyses; MO, AWW, and YS drafted the manuscript, and all authors read and approved its final version.

\section{Ethics approval and consent to participate}

The study was approved by the Ethical Committees of CSIC and the University Hospital Sant Joan and conducted in accordance with the 1975 Declaration of Helsinki revised in 1983. Written informed consent was obtained from the parents of the infants included in the study.

\section{Consent for publication}

Not applicable.

\section{Competing interests}

The authors declare that they have no competing interests.

\section{Publisher's Note}

Springer Nature remains neutral with regard to jurisdictional claims in published maps and institutional affiliations.

\section{Author details}

${ }^{1}$ Microbial Ecology, Nutrition and Health Research Unit, Institute of Agrochemistry and Food Technology, National Research Council (IATA-CSIC), C/Catedrático Agustín Escardino, 7. 46980, Paterna, Valencia, Spain. ²Gut Health Group, The Rowett Institute, University of Aberdeen, Aberdeen, UK. ${ }^{3}$ Genetics and Molecular Medicine Unit, Institute of Biomedicine of Valencia, National Research Council (IBV-CSIC), Valencia, Spain. ${ }^{4}$ Wellcome Trust Sanger Institute, Hinxton, Cambridgeshire, UK. ${ }^{5}$ Hospital Universitari de Sant Joan de Reus, IISPV, URV, Tarragona, Spain. ${ }^{6}$ Center for regenerative medicine, Boston university school of medicine, Boston, USA. Institut de Recerca Sant Joan de Déu and CIBERER, Hospital Sant Joan de Déu, Barcelona, Spain.

\section{Received: 30 October 2017 Accepted: 24 January 2018}

\section{Published online: 20 February 2018}

\section{References}

1. Husby S, et al. European Society for Pediatric Gastroenterology, Hepatology, and Nutrition guidelines for the diagnosis of coeliac disease. J Pediatr Gastroenterol Nutr. 2012;54(1):136-60.

2. Sollid LM. Coeliac disease: dissecting a complex inflammatory disorder. Nat Rev Immunol. 2002;2(9):647-55.

3. Dieli-Crimi R, Cenit MC, Nunez C. The genetics of celiac disease: a comprehensive review of clinical implications. J Autoimmun. 2015;64:26-41.

4. Romanos J, et al. Improving coeliac disease risk prediction by testing nonHLA variants additional to HLA variants. Gut. 2014;63(3):415-22.

5. Kuja-Halkola R, et al. Heritability of non-HLA genetics in coeliac disease: a population-based study in 107000 twins. Gut. 2016;65(11):1793-8.

6. Di Sabatino A, Corazza GR. Coeliac disease. Lancet. 2009;373(9673):1480-93.

7. Jabri B, Sollid LM. T cells in celiac disease. J Immunol. 2017;198(8):3005-14

8. Norris JM, et al. Risk of celiac disease autoimmunity and timing of gluten introduction in the diet of infants at increased risk of disease. JAMA. 2005; 293(19):2343-51.
9. Stordal K, White RA, Eggesbo M. Early feeding and risk of celiac disease in a prospective birth cohort. Pediatrics. 2013;132(5):e1202-9.

10. Vriezinga SL, et al. Randomized feeding intervention in infants at high risk for celiac disease. N Engl J Med. 2014;371(14):1304-15.

11. Lionetti $E$, et al. Introduction of gluten, HLA status, and the risk of celiac disease in children. N Engl J Med. 2014;371(14):1295-303.

12. Ivarsson A, et al. Breast-feeding protects against celiac disease. Am J Clin Nutr. 2002;75(5):914-21.

13. Sandberg-Bennich S, Dahlquist $G$, Kallen B. Coeliac disease is associated with intrauterine growth and neonatal infections. Acta Paediatr. 2002;91(1): 30-3.

14. Sanz Y, De Pama G, Laparra M. Unraveling the ties between celiac disease and intestinal microbiota. Int Rev Immunol. 2011;30(4):207-18.

15. Marild K, et al. Pregnancy outcome and risk of celiac disease in offspring: a nationwide case-control study. Gastroenterology. 2012;142(1):39-45. e3

16. Marild K, et al. Antibiotic exposure and the development of coeliac disease: a nationwide case-control study. BMC Gastroenterol. 2013;13:109.

17. Silano $M$, et al. Infant feeding and risk of developing celiac disease: a systematic review. BMJ Open. 2016;6(1):e009163.

18. Cenit MC, et al. Intestinal microbiota and celiac disease: cause, consequence or co-evolution? Nutrients. 2015;7(8):6900-23.

19. Collado $M C$, et al. Specific duodenal and faecal bacterial groups associated with paediatric coeliac disease. J Clin Pathol. 2009;62(3):264-9.

20. Wacklin $P$, et al. The duodenal microbiota composition of adult celiac disease patients is associated with the clinical manifestation of the disease. Inflamm Bowel Dis. 2013;19(5):934-41.

21. Wacklin $\mathrm{P}$, et al. Altered duodenal microbiota composition in celiac disease patients suffering from persistent symptoms on a long-term gluten-free diet. Am J Gastroenterol. 2014;109(12):1933-41.

22. Galipeau HJ, et al. Intestinal microbiota modulates gluten-induced immunopathology in humanized mice. Am J Pathol. 2015;185(11):2969-82.

23. Palma GD, et al. Influence of milk-feeding type and genetic risk of developing coeliac disease on intestinal microbiota of infants: the PROFICEL study. PLoS One. 2012;7(2):e30791.

24. Olivares $M$, et al. The HLA-DQ2 genotype selects for early intestinal microbiota composition in infants at high risk of developing coeliac disease. Gut. 2015;64(3):406-17

25. Frank $M$, et al. TLR signaling modulates side effects of anticancer therapy in the small intestine. J Immunol. 2015;194(4):1983-95.

26. Schloss PD, et al. Introducing mothur: open-source, platform-independent, community-supported software for describing and comparing microbial communities. Appl Environ Microbiol. 2009;75(23):7537-41.

27. White JR, Nagarajan N, Pop M. Statistical methods for detecting differentially abundant features in clinical metagenomic samples. PLoS Comput Biol. 2009;5(4):e1000352.

28. Segata N, et al. Metagenomic biomarker discovery and explanation. Genome Biol. 2011;12(6):R60.

29. Benjamini $Y$, Hochberg Y. Controlling the false discovery rate: a practical and powerful approach to multiple testing. J R Stat Soc Ser B Stat Methodol. 1995;57:289-300.

30. Palmer $\mathrm{C}$, et al. Development of the human infant intestinal microbiota. PLoS Biol. 2007;5(7):e177.

31. Koenig JE, et al. Succession of microbial consortia in the developing infant gut microbiome. Proc Natl Acad Sci U S A. 2011;108(Suppl 1):4578-85.

32. Backhed $F$, et al. Dynamics and stabilization of the human gut microbiome during the first year of life. Cell Host Microbe. 2015;17(5):690-703.

33. Cong $X$, et al. Gut microbiome developmental patterns in early life of preterm infants: impacts of feeding and gender. PLoS One. 2016;11(4): e0152751.

34. Sellitto $M$, et al. Proof of concept of microbiome-metabolome analysis and delayed gluten exposure on celiac disease autoimmunity in genetically at-risk infants. PLoS One. 2012;7(3):e33387.

35. Willing BP, et al. A pyrosequencing study in twins shows that gastrointestinal microbial profiles vary with inflammatory bowel disease phenotypes. Gastroenterology. 2010;139(6):1844-54. e1

36. Turnbaugh PJ, et al. A core gut microbiome in obese and lean twins. Nature. 2009;457(7228):480-4.

37. Chang JY, et al. Decreased diversity of the fecal microbiome in recurrent Clostridium difficile-associated diarrhea. J Infect Dis. 2008;197(3):435-8.

38. Kurilshikov A, et al. Host genetics and gut microbiome: challenges and perspectives. Trends Immunol. 2017;38(9):633-47. 
39. Brandtzaeg P. Update on mucosal immunoglobulin A in gastrointestinal disease. Curr Opin Gastroenterol. 2010;26(6):554-63.

40. Mathias $A$, et al. Role of secretory $\lg A$ in the mucosal sensing of commensal bacteria. Gut Microbes. 2014;5(6):688-95.

41. Mowat AM, et al. The role of dendritic cells in regulating mucosal immunity and tolerance. Novartis Found Symp. 2003;252:291-302. discussion 302-5

42. Mikulic J, et al. Secretory IgA in complex with Lactobacillus rhamnosus potentiates mucosal dendritic cell-mediated Treg cell differentiation via TLR regulatory proteins, RALDH2 and secretion of IL-10 and TGF-beta. Cell Mol Immunol. 2017;14(6):546-56.

Submit your next manuscript to BioMed Central and we will help you at every step:

- We accept pre-submission inquiries

- Our selector tool helps you to find the most relevant journal

- We provide round the clock customer support

- Convenient online submission

- Thorough peer review

- Inclusion in PubMed and all major indexing services

- Maximum visibility for your research

Submit your manuscript at www.biomedcentral.com/submit 